\title{
Planificación del transporte de madera rolliza en la industria forestal argentina
}

\section{Round wood transportation planning in the Argentine forest industry}

Presentación: 6-7/10/2020

\author{
Doctorando: \\ Maximiliano R. Bordón \\ Instituto de Desarrollo y Diseño (INGAR), Universidad Tecnológica Nacional (UTN) - Concejo Nacional de Investigaciones \\ Científicas y Tecnológicas (CONICET) - Argentina \\ mbordon@santafe-conicet.gov.ar
}

Director/es:

\section{Gabriela Corsano}

\section{Co-director/es:}

\section{Jorge M. Montagna}

\section{Resumen}

El sector forestal en Argentina es una de las principales fuentes de crecimiento para el país. Ha cobrado renovado interés en los últimos años debido a las oportunidades que ofrecen las actividades involucradas en su cadena de valor y el grado de desarrollo de la misma. El 95\% de la industria de base forestal del país se alimenta de madera rolliza cosechada en plantaciones forestales (es decir, bosques implantados). Estas plantaciones se concentran en mayor medida en la región noreste argentina (NEA), desde las cuales se abastece a los principales centros de consumo del país (Buenos Aires, Córdoba y Santa Fe). Dado que las distancias a cubrir entre los puntos de producción y de demanda son importantes, las actividades de logística forestal adquieren un papel determinante. El transporte de madera rolliza tiene un gran impacto en las estructuras de costos de las empresas, por lo que implementar mejoras en la planificación de esta actividad se traduce en un ahorro significativo para las empresas del sector. En base a esta problemática se han desarrollado modelos de programación matemática y algoritmos heurísticos enfocados en la resolución eficiente de problemas asociados a la planificación del transporte de madera rolliza. En este trabajo se presentan los principales resultados y avances de la tesis doctoral titulada "Modelos de optimización y estrategias de resolución para la gestión de la logística de abastecimiento y distribución de los recursos forestales". Se describen brevemente las características de los problemas abordados, la metodología desarrollada para resolverlos y los principales resultados obtenidos.

Palabras clave: Logística forestal, Programación matemática, Ruteo de vehículos, Scheduling.

\footnotetext{
Abstract

The forestry sector in Argentina is one of the main sources of growth in the country. It has gained renewed interest in recent years due to the opportunities offered by the activities involved in its value chain and its degree of development. $95 \%$ of the forest-based industry in the country is fed by round wood harvested in forest plantations (that is, planted forests). These plantations are mainly concentrated in the northeast region of Argentina (NEA), from where the main consumption centers of the country (Buenos Aires, Córdoba and Santa Fe) are supplied. Since the distances to be covered between production and demand nodes are important, the forestry logistics activities acquire a decisive role. The transportation of round wood has a great impact on the cost structures of companies, so implementing improvements in the planning of this activity translates into significant savings for companies in the sector. Based on this problem, mathematical programming models and heuristic algorithms have been developed focused on the efficient resolution of problems associated with planning the transportation of round wood. This
} 
paper presents the main results and advances of the doctoral thesis entitled "Modelos de optimización y estrategias de resolución para la gestión de la logística de abastecimiento y distribución de los recursos forestales". The characteristics of the addressed problems, the methodology developed to solve them and the main results obtained are briefly described.

Keywords: Forestry logistics, Mathematical programming, Vehicle routing, Scheduling.

\section{Introducción}

En los últimos 20 años se evidenció una tendencia creciente en los niveles de extracción de madera rolliza en bosques implantados (Ministerio de Agricultura, Ganadería y Pesca, 2018). Este crecimiento en cuanto a producción de madera rolliza se debe principalmente a que las industrias de base forestal representan una parte importante del entramado industrial nacional. Una gran parte de los productos obtenidos en la etapa de producción primaria (la cual se alimenta de madera rolliza) son utilizados como materia prima por otras industrias (producción secundaria), como por ejemplo tableros de fibra y tableros de partículas para la construcción, tableros compensados para molduras, pasta celulósica para la elaboración de papel, o incluso aserrín y subproductos para la generación de energía. En este sentido, los bosques nativos e implantados tienen un rol determinante como fuentes de materia prima forestal.

El problema de la logística forestal presenta muchas variantes y depende de las particularidades de cada empresa y región (Audy y col, 2012). Los aspectos que se consideran en este trabajo son los más elementales, pero si se combinan dan una idea del amplio espectro de modelos que se requiere para abordar este problema y de las dificultades para plantear un enfoque único que permita representarlo (Bordón y col, 2019). Entre estos aspectos se destacan: 1) flota de camiones: fija o variable, de características homogéneas o heterogéneas (de acuerdo a si poseen o no distintas capacidades de carga), alojados en depósito único o en diversas bases regionales, diferentes condiciones operativas (tiempos de operación, cantidad de viajes o ciclos a realizar, etc.) y organizacionales (flota perteneciente a un mismo dueño o cooperativas de trabajo, etc.); 2) tipo de viaje: camión parcialmente cargado o viaje a carga completa, transporte de una única materia prima por viaje o una variedad de las mismas; 3 ) horizonte de planificación, yendo desde lo diario a lo semanal; 4) decisiones referidas a la programación de arribos de camiones, ya sea en el origen como en el destino de la materia prima; y 5) objetivo perseguido: basado en el costo (por distancia recorrida con y sin carga, por utilización de un vehículo, entre otros), basado en el tiempo (tiempos ociosos o de espera, tardanza máxima, entre otros), u otras medidas de performance (un mix entre los anteriores, nivel de servicio al cliente, entre otras).

Si bien el listado anterior no es exhaustivo, se pueden apreciar las diversas variantes asociadas a un mismo problema base y la dificultad a la hora de abordar todas estas variantes en un único enfoque. A pesar de esto, en el contexto argentino, la implementación de sistemas de soporte a las decisiones en el sector forestal es relativamente escasa, por lo que resulta necesario abordarlas (Broz y col., 2018).

Se destacan tres problemas importantes relacionados con las actividades de transporte: el problema de asignación de materia prima, el problema de ruteo de vehículos y el problema de programación (o scheduling) de arribos. El problema de asignación de materia prima se relaciona con el establecimiento de los niveles de abastecimiento de materia prima desde cada bosque o rodal hacia cada planta (es decir, el flujo de materia prima entre cada punto de la red). El problema de ruteo de vehículos se relaciona con la secuencia de visitas que debe realizar cada camión en cada punto de red. Por su parte, el problema de scheduling de arribos se relaciona con la secuenciación de las llegadas de los camiones en cada nodo de la red de forma tal de evitar superposiciones entre los mismos, en otras palabras, con el establecimiento de tiempos específicos para cada actividad (carga/descarga de camión). Estos problemas estrechamente relacionados usualmente suelen abordarse de manera desacoplada en la literatura, por lo que el objetivo de la tesis doctoral es abordarlos de forma eficiente. En la sección "Desarrollo" se detallan los trabajos desarrollados en el área, caracterizándolos en base a las alternativas antes planteadas, destacando las virtudes y debilidades de cada uno (por cuestiones de espacio no se presentan los modelos matemáticos desarrollados), mientras que en la sección "Resultados" se presentan los principales resultados obtenidos.

\section{Desarrollo}


En el primero de los trabajos realizados (Bordón y col., 2018) se aborda la problemática de asignación de materia prima y ruteo de vehículos. El modelo de programación matemática mixto entero lineal (MILP, de sus siglas en inglés) desarrollado abarca el horizonte de planificación diario, con el objetivo de generar los recorridos de cada camión a costo mínimo (compuesto por costos por kilómetro recorrido con y sin carga, y costos fijos por utilización de camión), mientras se garantiza el suministro de materia prima rolliza. Los modelos matemáticos que usualmente se encuentran en la literatura asumen que los viajes (o entregas) a realizar se conocen de antemano o se generan en una etapa previa al ruteo de vehículos. A diferencia de esos enfoques, en este trabajo se abordan ambas decisiones en conjunto (asignación y ruteo de vehículos). Se asume que los camiones (flota homogénea) realizan viajes a carga completa. Se considera además que cada camión se aloja en una base regional desde la que inicia el recorrido y a la que debe regresar luego de completar todos los viajes. El modelo MILP propuesto es analizado a través de un conjunto de escenarios, resolviendo eficientemente el problema abordado en todos ellos en un tiempo de cómputo reducido. En la Tabla 1 (ver sección "Resultados") se presentan los resultados obtenidos. Este trabajo se corresponde con la referencia (T1).

En el trabajo mencionado previamente (T1) se asume que cada planta demanda un único tipo de materia prima (por ejemplo, madera rolliza con destino aserrable o pulpable). Este enfoque es válido incluso cuando lo que se traslada es el fuste completo sin trozar. Sin embargo, cuando se requiere de mayor precisión en cuanto al tipo de materia prima transportada, este enfoque resulta insuficiente. Para abordar esta situación, se desarrolló un modelo MILP en donde se asume que cada planta (o cliente) requiere diferentes tipos de materias primas, ampliando el alcance del trabajo presentado previamente. De esta manera, el suministro de materias primas específicas es otra variable de decisión a tener en cuenta. Estos supuestos aumentan significativamente la complejidad del modelo. Sin embargo, dado que para problemas de tamaño industrial el tamaño del modelo (en cuanto a cantidad de variables y restricciones) es importante, se propuso un enfoque de resolución basado en generación de columnas (GC) para abordarlo. Este enfoque permite encontrar soluciones cercanas al óptimo en tiempos de resolución extremadamente inferiores. En la Tabla 1 se presentan los resultados obtenidos, bajo la referencia (T2).

En los enfoques presentados en los trabajos previos, (T1) y (T2), no se consideran las decisiones referidas a la programación de arribos de los camiones en cada nodo de la red. Para abordar este aspecto, en Bordón y col. (2020) se presenta un modelo MILP que considera en forma simultánea las decisiones de asignación de materias primas, ruteo de vehículos y programación de arribos, extendiendo el alcance de los modelos presentados en los trabajos previos. Dado que para problemas de gran envergadura los tiempos de resolución no son lo suficientemente competitivos como para transferir el modelo directamente a la práctica, se presenta un enfoque de resolución secuencial en donde en una primera etapa se resuelven los problemas de asignación de materia prima y ruteo de vehículos para posteriormente resolver el problema de scheduling de arribos. Los resultados obtenidos son presentados en la Tabla 1, bajo la referencia (T3).

El enfoque secuencial presentado en Bordón y col. (2020) puede dar lugar a soluciones no factibles ya que en la primera etapa se podrían obtener recorridos que no puedan ser programados dentro de las ventanas de tiempo correspondientes (en este caso, los camiones tienen limitada su jornada laboral y no se permiten excesos en la misma -horas extras-). En base a esto, se desarrolló un enfoque de resolución iterativo en el que se incluye la metodología presentada en Bordón y col. (2020). Básicamente, en la primera etapa se resuelven los problemas de asignación de materia prima y ruteo de vehículos para luego, en la segunda etapa, determinar la programación de arribos considerando las rutas obtenidas en la etapa previa. Si en la segunda etapa ningún camión se excede del tiempo máximo de estadía en ruta permitido, el algoritmo finaliza; en caso contrario, se agrega una restricción de corte para excluir del espacio de búsqueda la solución obtenida en la primera etapa, y se ejecuta una nueva iteración. El algoritmo termina cuando ningún camión se excede de las horas establecidas o cuando se alcanza el máximo número de iteraciones permitido, entregando la solución con menor exceso encontrada hasta el momento. Los resultados obtenidos se presentan en la Tabla 1, bajo la referencia (T4).

En la siguiente sección se presentan resumidamente los problemas resueltos a través los trabajos (T1) a (T4) y se detallan los tiempos de resolución correspondientes.

\section{Resultados}


En esta sección se presentan los principales resultados de cada una de las metodologías brevemente descriptas en la sección previa (trabajos T1 a T4). La Tabla 1 que se presenta a continuación incluye las características de cada uno de los problemas abordados. En dicha tabla se observa el tipo de problema que se está resolviendo (asignación: A, ruteo: $\mathrm{R}$, scheduling: $\mathrm{S}$ ), la cantidad de camiones que se utilizan en la solución final (\#C), la cantidad de bases regionales consideradas (\#P), la cantidad de plantas o clientes (\#I), la cantidad de materias primas (\#MP), la cantidad de bosques (\#F), la demanda total a satisfacer (en cargas completas de camión), la metodología de resolución empleada (exacta: E, heurística: H), y el tiempo de resolución (en segundos).

\begin{tabular}{|c|c|c|c|c|c|c|c|c|c|}
\hline Trabajo & $\begin{array}{c}\text { Tipo } \\
\text { problema }\end{array}$ & \#C & \#P & \#I & \# MP & \#F & $\begin{array}{c}\text { Demanda } \\
\text { total }\end{array}$ & $\begin{array}{c}\text { Metodología } \\
\text { resolución }\end{array}$ & $\begin{array}{c}\text { Tiempo } \\
\text { resolución(s) }\end{array}$ \\
\hline $\mathrm{T} 1$ & $\mathrm{~A} / \mathrm{R}$ & 250 & 12 & 6 & 3 & 15 & 750 & $\mathrm{E}$ & 247,76 \\
\hline $\mathrm{T} 2$ & $\mathrm{~A} / \mathrm{R}$ & 250 & 12 & 5 & 5 & 15 & 750 & $\mathrm{E} / \mathrm{H}$ & 122,95 \\
\hline $\mathrm{T} 3$ & $\mathrm{~A} / \mathrm{R} / \mathrm{S}$ & 16 & 5 & 3 & 2 & 10 & 47 & $\mathrm{E} / \mathrm{H}$ & 932,01 \\
\hline $\mathrm{T} 4$ & $\mathrm{~A} / \mathrm{R} / \mathrm{S}$ & 17 & 3 & 3 & 2 & 7 & 51 & $\mathrm{E} / \mathrm{H}$ & 39,71 \\
\hline
\end{tabular}

Tabla 1: caracterización de los problemas abordados

En la tabla anterior puede observarse que los tiempos de resolución correspondientes a los modelos que abordan la problemática de asignación de materia prima y ruteo de vehículos en conjunto son más que interesantes. Sin embargo, al incorporar los aspectos relacionados a las decisiones de programación de arribos de camiones el problema se hace más difícil de resolver y, en consecuencia, los tiempos de resolución empeoran. A pesar de esto, a través de los modelos matemáticos desarrollados y los enfoques de resolución propuestos se logra dar respuesta a problemas de tamaño importante en tiempos de resolución competentes.

En el planeamiento de las operaciones de transporte en la industria forestal es importante poder balancear los suministros y demandas entre los distintos períodos de tiempo para lograr estabilidad en la producción, distribución y comercialización de los productos involucrados. Una coordinación estrecha entre lo que se cosecha y lo que efectivamente se requiere en los puntos de demanda no sólo representa una mejora en la eficiencia productiva sino que también significa un ahorro importante en costos, dado que la materia prima cosechada puede perder valor comercial si no es transportada y procesada en el tiempo debido. En la literatura existente los problemas de corte transversal de troncos (bucking, del inglés) y distribución de troncos son abordados de forma desacoplada, es decir, primero se planifica la cosecha y posteriormente se planifica el transporte, con las consecuencias que esto acarrea (utilización de recursos de forma ineficiente). El último de los trabajos que componen la tesis doctoral (trabajo actualmente en desarrollo) aborda este gap en la literatura, en donde se desarrolla un modelo MILP que considera simultáneamente las operaciones de cosecha y transporte, para un horizonte de planificación semanal. Más precisamente, el modelo propuesto permite determinar en qué período se realizarán las actividades de corte de fustes en los bosques, qué patrones de corte se utilizarán, qué cantidad de troncos de determinado largo y diámetro se generarán en consecuencia, qué niveles de inventario de troncos se mantendrán (tanto en bosques como en plantas), qué tamaño de flota será necesario considerar para realizar la distribución, cómo estará conformada la carga de cada viaje a realizar por cada camión, y cuál será el recorrido que realizará cada camión.

En la industria forestal, el costo asociado al transporte de materia prima rolliza tiene un gran impacto en las estructuras de costos de las empresas del sector, por lo que reducirlos implicaría un ahorro importante. Un suministro confiable de materia prima rolliza que permita la disponibilidad de la madera adecuada en el momento adecuado, en el lugar adecuado, es una ventaja competitiva. En el contexto argentino, las empresas generalmente toman decisiones sobre la distribución de troncos en base a su experiencia, es decir, soluciones heurísticas. Por lo tanto, el uso de una herramienta de planificación para apoyar estas decisiones redundaría en mejores resultados. Los resultados obtenidos a través de los modelos matemáticos desarrollados en los trabajos expuestos previamente demuestran capacidad para abordar de manera eficiente y efectiva el problema de distribución de los recursos forestales. 


\section{Referencias}

Audy, J.-F., D’Amours, S., Rönnqvist, M. (2012). Planning methods and decision support systems in vehicle routing problems for timber transportation: A review. CIRRELT, 38. https://www.cirrelt.ca/DocumentsTravail/CIRRELT2012-38.pdf (último acceso Agosto 2020).

Broz, D., Rossit, D., Rossit, D., Cavallin, C. (2018). The Argentinian forest sector: opportunities and challenges in supply chain management.Uncertain Supply Chain Management, 6(4), 375-392. doi:10.5267/j.uscm.2018.1.001.

Bordón, M.R., Montagna, J.M., Corsano, G. (2018). An exact mathematical formulation for the optimal log transportation. Forest Policy and Economics. 95, 115-122. doi:10.1016/j.forpol.2018.07.017.

Bordón, M.R.; Montagna, J.M.; Corsano, G. (2019). Planificación del transporte en la industria forestal argentina: aplicaciones basadas en programación matemática. XIII Jornadas Técnicas Forestales y Ambientales (JOTEFA). Octubre de 2019. Misiones, Argentina.

Bordón, M.R., Montagna, J.M., Corsano, G. (2020). Mixed integer linear programming approaches for solving the raw material allocation, routing and scheduling problems in the forest industry. International Journal of Industrial Engineering Computations. 11(4), 525-548. doi:10.5267/j.ijiec.2020.5.001.

Ministerio de Agricultura, Ganadería y Pesca (2018). Sector Forestal 2017.

https://www.magyp.gob.ar/sitio/areas/ss_desarrollo_foresto_industrial/estadisticas/_archivos//oooooo_Sector\%2 oForestal/oooooo_Informes/170000_2017\%20-\%20Sector\%20Forestal.pdf (último acceso Agosto 2020). 